\title{
CHORIOCARCINOMA OF THE OVARY: A CASE REPORT
}

\section{Ekanayake $\mathrm{CD}^{1}$, Wijesinghe $\mathrm{PS}^{2}$, Herath $\mathrm{RP}^{2}$, Puliyadda TMNK${ }^{2}$, Hewavisenthi SJdeS}

\section{Abstract}

Ovarian choriocarcinoma whether gestational or non-gestational, is an extremely rare malignant germ cell tumour. We report a young patient with an ovarian choriocarcinoma who was successfully treated with fertility sparing conservative surgery and adjuvant chemotherapy.

\section{INTRODUCTION}

Ovarian choriocarcinoma is an extremely rare tumour which may be gestational or non-gestational in origin. Gestational ovarian choriocarcinoma arise from an ovarian ectopic pregnancy or as a metastasis from uterine or tubal choriocarcinoma, and has an estimated incidence of 1 in 369 million pregnancies ${ }^{1}$. Nongestational ovarian choriocarcinoma itself is rare and accounts for less than $0.6 \%$ of all ovarian neoplasms ${ }^{2}$. Due to its rarity, information on the clinicopathological features, diagnosis, and therapeutic options are limited, and this poses a dilemma in the management, as most of these patients are in the reproductive age group with intentions of future fertility.

\section{CASE REPORT}

A 20 year old woman with oligomenorrhoeic cycles presented with a positive urine pregnancy test at a period of amenorrhoea of five weeks. On examination there was a large abdomino-pelvic mass extending

${ }^{1}$ Professorial Obstetrics and Gynaecology Unit, North Colombo Teaching Hospital, Ragama

${ }^{2}$ Department of Obstetrics and Gynaecology, Faculty of Medicine, University of Kelaniya

${ }^{3}$ Department of Pathology, Faculty of Medicine, University of Kelaniya

Correspondence: Ekanayake CD

No 54 Pushpadana Mw, Kandy, Sri Lanka

E-mail: cdekanayake2000@yahoo.com,

Competing interests -none

Patient was treated at the Professorial Obstetrics and Gynaecology Unit, NCTH, Ragama. up to the umbilicus. The uterus was normal in size with a solid left adnexal mass and a cystic right adnexal mass. Laboratory investigations revealed serum $\beta$ hCG $>1 \times 10^{6} \mathrm{mIU} / \mathrm{ml}$, AFP $1 \mathrm{ng} / \mathrm{ml}, \mathrm{CA}-125100 \mathrm{U} / \mathrm{ml}$. Ultrasonography revealed a left sided $7.5 \times 7.2 \mathrm{~cm}$ solid ovarian tumour and a $15 \times 10 \mathrm{~cm}$ right sided multilocular ovarian cyst with no ascites. During staging laparotomy, a haemorrhagic solid ovarian tumour measuring $8 \times 8 \mathrm{~cm}$ on left side (Figure 1, 2), a multilocular ovarian cyst measuring $15 \times 10 \mathrm{~cm}$ on the right side (Figure 3) and small amount of free fluid were found. A left salpingo-oophorectomy and an infracolic omentectomy were done. The multilocular cyst on the right side was thought to be a theca lutein cyst and was left undisturbed. Histology revealed a choriocarcinoma in the left ovary exhibiting syncytiotrophoblastic and cytotrotrophoblastic cells growing in

Figure 1 - A solid ovarian mass arising from left ovary.

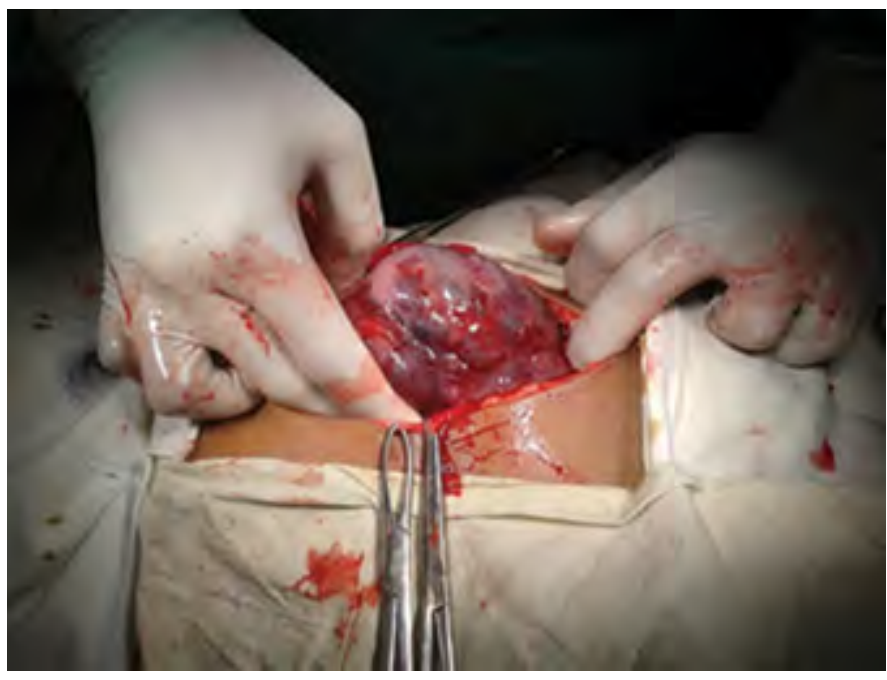

Figure 2 - Cut surface of solid ovarian mass showing necrotic and haemorrhagic areas.

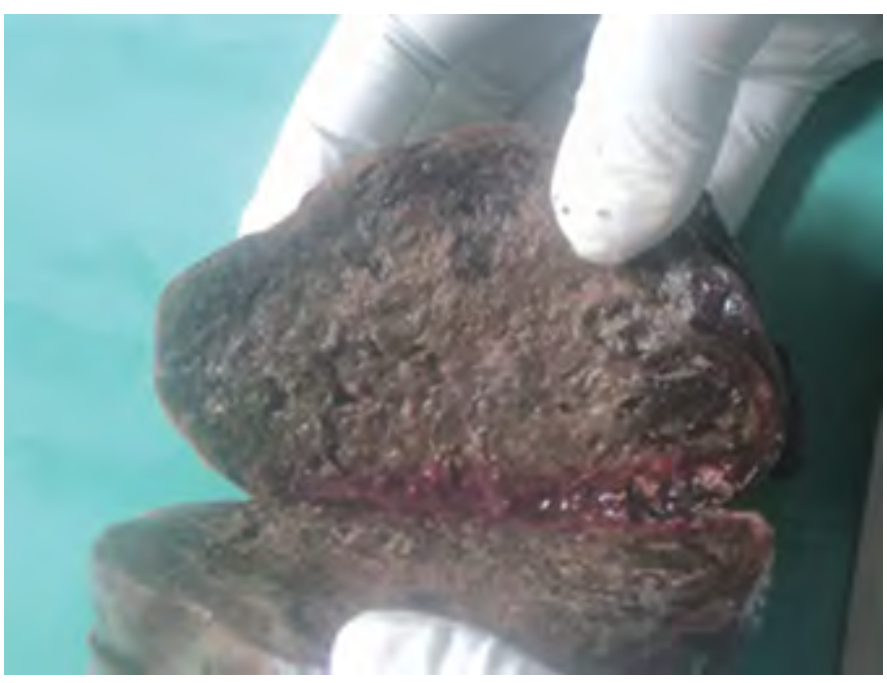


Figure 3 - Right multilocular cyst theca lutein cyst.

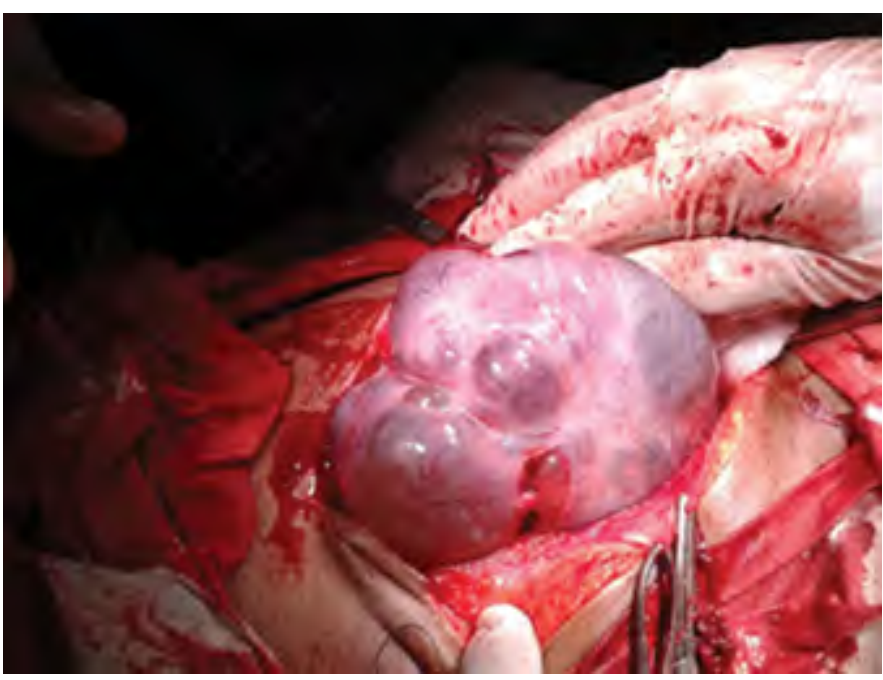

a plexiform pattern. Suction curettage at the time of laparotomy did not reveal any products of conception. It was considered to be a FIGO stage 1a ovarian choriocarcinoma. The patient responded well to chemotherapy with EMACO regimen (etoposide, methotrexate, actinomycin D, cyclosphosphamide and vincristine), with serum $\beta$ hCG falling to 0.96 $\mathrm{mIU} / \mathrm{ml}$ and imaging confirming the regression of the theca lutein cyst. One year after the completion of the treatment, she is asymptomatic and having regular cycles with normal pelvic imaging and an undetectable serum $\beta$ hCG level.

\section{DISCUSSION}

The differential diagnosis of a solid abdomino-pelvic mass with markedly elevated serum $\beta$ hCG includes; mixed germ cell tumour of the ovary, pure non-gestational ovarian choriocarcinoma, gestational ovarian choriocarcinoma following an ovarian ectopic pregnancy or an ovarian metastasis from uterine or tubal choriocarcinoma.

The absence of other cell lines on histology and a normal AFP excluded a mixed germ cell tumour. Absence of products of conception at suction curettage excluded a uterine choriocarcinoma. The presence of a histologically normal left tube and a macroscopically normal right tube excluded the rare possibility of a tubal choriocarcinoma.

Therefore the possibilities were either a pure non-gestational ovarian choriocarcinoma or a gestational ovarian choriocarcinoma. Conventional histopathology is not able to distinguish between the two and molecular genetic analysis to demonstrate the presence of paternal antigens in gestational choriocarcinoma is needed for a definitive diagnosis ${ }^{3,4}$. The presence of oligomenorrhoeic cycles may give an impression that this patient may have had a period of amenorrhoea which favours a tumour of gestational origin. Non-gestational choriocarcinomas are commoner in the premenarcheal age group and therefore the possibility of a gestational choriocarcinoma has to be considered in this patient.

Germ cell tumours tend to occur mainly in young adults and children and therefore fertility conserving treatment is a basic necessity in the care of these individuals. Currently conservative surgery is the standard treatment for stage 1 germ cell tumours ${ }^{5}$. Even in those who require chemotherapy, the majority generally resume their normal menstrual function after chemotherapy.
There are no standard chemotherapeutic regimens for ovarian choriocarcinoma due to the low incidence of these tumours. Gestational choriocarcinoma generally responds to methotrexatebased chemotherapy. However nongestational ovarian choriocarcinoma has a poorer prognosis and may be resistant to single agent therapy. As we did not have the facilities to differentiate between the two entities in our patient, she was treated with the EMACO regimen with a good outcome.

\section{CONCLUSION}

Ovarian chriocarcinoma is an extremely rare germ cell tumour which poses a therapeutic challenge as most patients are in the reproductive age group with intentions of future fertility. In such circumstances fertility conserving treatment must be considered which must to be weighed against the risk of recurrence in a given case.

\section{REFERENCES}

1. Axe SR, Klein VR, Woodruff JD. Choriocarcinoma of the ovary. Obstetrics \& Gynecology 1985; 66: 111-4.

2. Vance RP, Geisinger KR. Pure nongestational choriocarcinoma of the ovary: report of a case. Cancer 1985; 56 : 2321-5.

3. Lv L, Yang $\mathrm{K}, \mathrm{Wu} \mathrm{H}$ et al. Pure choriocarcinoma of the ovary: a case report. Journal of Gynecologic Oncology 2011; 22(2): 135-9.

4. Yamamoto E, Ino K, Yamamoto $T$, et al. A pure nongestational choriocarcinoma of the ovary diagnosed with short tandem repeat analysis: case report and review of the literature. International Journal of Gynecological Cancer 2007; 17: 254-8.

5. Ellis $P$, Mould T. Fertility-saving treatment in gynaecological oncology. The Obstetrician \& Gynaecologist 2009;11: 239-4. 